\title{
Atitudes e conhecimento dos pais quanto ao uso de dentifrícios fluoretados em crianças de um a 65 meses de idade
}

\author{
Attitudes and knowledge of parents regarding the use of fluoride \\ dentifrices in children aged one to sixty-five months
}

\author{
Núbia Rosa Prietto* \\ Andressa Rockenbach Portela** \\ Luiza Helena Almeida*** \\ Anna Paula da Rosa Possebon* \\ Marina Sousa Azevedo ${ }^{* * * *}$ \\ Dione Dias Torriani ${ }^{* * *}$
}

\section{Resumo}

Objetivo: analisar o conhecimento dos pais sobre o flúor, os dentifrícios, seu uso e o motivo da escolha. Sujeitos e método: estudo transversal com dados coletados em entrevistas com pais de crianças de um a 65 meses de idade, sobre questões socioeconômicas, conhecimento sobre flúor, motivo pela escolha do dentifrício, quantidade utilizada e hábitos de higiene bucal da criança. Os dados foram analisados por meio de estatística descritiva. Resultados: foram entrevistados 202 pais. A média de idade das crianças participantes do estudo foi de 35,6 meses $( \pm 15,06)$. Os pais citaram os seguintes critérios para a compra do dentifrício: ausência de flúor $(20,3 \%)$, preço $(6,9 \%)$, sabor $(11,4 \%)$, marca $(10,9 \%)$, e $20,8 \%$ dos pais responderam não haver critério. Com relação ao início da administração do dentifrício fluoretado para as crianças, $43,1 \%$ dos pais relataram que ocorreu até um ano, 21,8\% mais de um ano, e $35,1 \%$ não lembravam. Sobre a quantidade utilizada de dentifrício, 32,2\% dos pais apontaram usar a técnica longitudinal, 41,1\% a transversal, 26,2\% a do grão de arroz, e 5\% não responderam. Com relação à higiene bucal ser realizada sozinha ou com ajuda, em $12,2 \%$ ela é realizada somente pela criança, em $47,7 \%$ dos casos é a criança com a ajuda de um adulto, e em $40,1 \%$ é feita somente pelo adulto. Conclusão: Os pais têm conhecimentos sobre o flúor e sua finalidade, porém não utilizam a dose recomendada para a idade e não há uma conscientização quanto à motivação para a escolha do dentifrício. Assim, há necessidade de investir em educação a respeito dos dentifrícios para a escolha consciente e o uso adequado.

Palavras-chave: Flúor. Pré-escolar. Dentifrícios.

\section{Introdução}

Os responsáveis pela saúde pública brasileira, no que se refere à saúde bucal, tem enfatizado a necessidade de aumentar os conhecimentos educativos, a fluoretação das águas de abastecimento público e orientação quanto à indicação dos dentifrícios fluoretados com intuito de controlar e prevenir a cárie dentária ${ }^{1}$. Apesar de a prevalência da cárie do Sul, Brasil.

*** Cirurgiã-dentista, Faculdade de Odontologia, Universidade Federal de Pelotas, Pelotas, Rio Grande do Sul, Brasil.

Professora, Departamento de Odontologia Social e Preventiva, Programa de Pós-Graduação em Odontologia, Faculdade de Odontologia, Universidade Federal de Pelotas, Pelotas, Rio Grande do Sul, Brasil. 
estar declinando, ela ainda é considerada um relevante problema de saúde pública. De acordo com os dados do SB Brasil de 2010, o índice de dentes ceo-d (superfícies cariadas, extração indicada, obturadas) em crianças de 5 anos de idade passou de 2,8 , em 2003, para 2,3 , em 2010, uma redução de apenas $17 \%$, com $80 \%$ desses dentes não tratados ${ }^{2}$.

A utilização de flúor na prevenção e no controle da cárie é uma das medidas de saúde pública mais estudadas $^{3-5}$. Atualmente, uma das formas mais amplas da utilização do flúor é o emprego de dentifrícios fluoretados associados à escovação mecânica ${ }^{4}$. De acordo com as novas recomendações da comunidade científica, a concentração de flúor a ser administrada nas escovas dentais é de 1.100 ppm de F, em pequenas doses, de acordo com a faixa etária da criança ${ }^{4,6,7}$. O flúor utilizado na composição do dentifrício possui tanto um papel preventivo, por ser capaz de reduzir a perda de minerais do esmalte do dente hígido, como também terapêutico, pois aumenta a reposição desses no dente com lesão de cárie ${ }^{8}$.

Entretanto, o uso de dentifrícios fluoretados em crianças mais jovens deve ser supervisionado pelos pais, uma vez que elas tendem a engolir de $30 \%$ a $63,2 \%$ do produto durante a escovação $0^{9-12}$. Entre os fatores de ingestão de dentifrícios, está o fato de a criança não ter o reflexo de expectoração totalmente desenvolvido ${ }^{13}$. Sendo assim, esse cuidado deve ser considerado principalmente entre idades pré-escolares, pois é o período de calcificações das coroas dentárias ${ }^{14} \mathrm{e}$, se não controlado, pode levar à fluorose dentária na dentição permanente ${ }^{13,15}$.

A literatura deixa claro que os pais conhecem amplamente os benefícios do flúor na proteção dos dentes contra a cárie dentária. No entanto, o conhecimento sobre a relação de excesso de fluoretos e fluorose dentária entre os pais é pouco difundi$\mathrm{do}^{16-18}$. Somado a isso, os pais parecem ter dificuldades durante a escolha do produto, devido à grande variedade de dentifrícios exclusivos para crianças no mercado ${ }^{19}$. Os dentifrícios fluoretados, principalmente os infantis, utilizam estratégias publicitárias para atrair as crianças e estimular o consumo, e tais estratégias superam os recursos educativos ${ }^{20}$.

Para tanto, é notório investigar as orientações dos pais sobre a administração desse produto. Assim, o objetivo deste trabalho foi analisar o conhecimento dos pais sobre os dentifrícios e a presença de flúor nesses produtos e sua finalidade, a forma de uso, assim como a motivação para a escolha do dentifrício para a criança.

\section{Sujeitos e método}

Este estudo foi aprovado pelo Comitê de Ética e Pesquisa da Faculdade de Odontologia da Universidade Federal de Pelotas sob o Parecer no 24/2012, atendendo aos requisitos da resolução do Conselho Nacional de Saúde, CNS nº 466/96.
Trata-se de um estudo transversal, com uma amostra de conveniência, realizado no maior supermercado central do município de Pelotas, no estado do Rio Grande do Sul, no ano de 2013. A população da cidade, segundo dados do IBGE no ano de 2010 é de 320 mil habitantes, com fluoretação da água de abastecimento desde 1961, com concentração de flúor que varia de 0,6 a $0,9 \mathrm{mgF} / \mathrm{L}^{21}$. O estudo foi conduzido com pais e mães de crianças de 1 a 65 meses de idade, escolhidos aleatoriamente em um supermercado da cidade. A escolha pelo supermercado foi motivada pelo fato de ser o maior supermercado na área central na cidade, e, até o final de 2013 (antes da instalação do shopping na cidade), era o lugar de referência para compras de diversos utensílios domésticos, eletrônicos e alimentícios, por isso bastante frequentado por pessoas de distintos bairros e com perfis de todas as classes sociais. Após prévia autorização do responsável pelo estabelecimento comercial, todos os pais que entraram no supermercado acompanhados de filhos com idade entre um e 65 meses de idade, foram convidados a participar do estudo. Todos assinaram um termo de consentimento livre e esclarecido.

O levantamento de dados consistiu em uma entrevista com os pais realizada por meio de um questionário semiestruturado. Para a entrevista, duas alunas da Faculdade de Odontologia foram previamente treinadas em uma entrevista piloto realizada com pais de crianças que frequentam a unidade de clínica infantil da instituição de ensino. Além de promover o treinamento, a ação serviu para testar o questionário elaborado.

O questionário foi dividido em três partes, a primeira parte com questões socioeconômicas informativas sobre a criança (idade), a segunda continha dados sobre o conhecimento dos pais sobre flúor e os dentifrícios, quanto ao motivo pela escolha e quantidade utilizada, e na terceira parte, os hábitos de higiene bucal da criança.

Para as questões socioeconômicas foram coletadas as variáveis renda e escolaridade materna. A variável renda foi coletada na forma contínua e categorizada em tercis, sendo o primeiro tercil considerado de $\mathrm{R} \$ 750,00$ a $\mathrm{R} \$ 2.000,00$, o segundo tercil de $R \$ 2.001,00$ a $R \$ 3.400,00$, e o terceiro tercil de $R \$ 3.401,00$ a $R \$ 16.000,00$. Enquanto que a variável escolaridade materna foi coletada na forma contínua em anos de estudo, e categorizada em: de zero a oito, nove a onze e doze ou mais anos de estudo. Para a variável idade da criança, foi realizada coleta na forma contínua, e categorizada da seguinte forma: zero a doze, treze a 24,25 a 36,37 a 48,49 a 60 e 61 a 65 meses de idade.

As perguntas relacionadas à saúde bucal foram divididas de forma que abordassem aspectos como: a) hábitos de higiene da criança: idade que começou a usar pasta de dente com flúor, quem faz a higiene bucal da criança, quem coloca pasta de dente na escova da criança; b) critérios de escolha do creme 
dental: marca, indicação, presença de flúor, preço e sabor;.c) conhecimento sobre o flúor: para que serve, qual a quantidade de flúor na pasta adquirida e se houve recomendação para o uso de pasta sem flúor.

Ao final da entrevista, foi mostrado um folheto contendo fotos da quantidade de dentifrício a ser colocada na escova (grão de arroz, técnica transversal e técnica longitudinal), a fim de que o responsável pela higiene bucal da criança indicasse a foto que melhor representasse a quantidade normalmente colocada. Em nenhum momento expressou-se de forma oral a quantidade: pequena ou do tamanho de um grão de ervilha ou de grão de arroz, para não induzir a resposta, uma vez que esses parâmetros são subjetivos e, portanto, podem variar entre os indivíduos.

Os dados coletados foram tabulados em planilha eletrônica Microsoft Excel e transferida para o software Stata versão 9.1 (Stata Corporation, College Station, TX, EUA), para a análise descritiva com distribuição das frequências relativa e absoluta entre as variáveis coletadas.

\section{Resultados}

Foram entrevistados 202 pais, cujas crianças tinham entre um e 65 meses, com média de idade de 35,6 meses $( \pm 15,06)$. Apenas um sujeito se recusou a responder ao questionário. Com padrão mínimo de $R \$ 750,00$ e $R \$ 16.000,00$ como teto máximo, a renda média familiar dos entrevistados foi de $R \$$ $3.194,30 \pm 2.164,80$. A Tabela 1 apresenta a distribuição da amostra de acordo com os dados socioeconômicos e a idade das crianças.

Tabela 1 - Distribuição da amostra segundo características socioeconômicas, Pelotas, 2013 ( $n=202)$

\begin{tabular}{|c|c|c|}
\hline Variáveis & $\mathrm{N}$ & $\%$ \\
\hline Renda familiar (em tercil)* & & \\
\hline $1^{\circ}$ tercil $(\mathrm{R} \$ 750-2000)$ & 69 & 35,6 \\
\hline $2^{\circ}$ tercil $(\mathrm{R} \$ 2001-3400)$ & 63 & 32,4 \\
\hline $3^{\circ}$ tercil (R\$ 3401-16000) & 62 & 32,0 \\
\hline Escolaridade materna (em anos de estudo) & & \\
\hline $0-8$ & 24 & 11,9 \\
\hline $9-11$ & 64 & 31,7 \\
\hline$\geq 12$ & 114 & 56,4 \\
\hline Idade das crianças por faixa em meses** & & \\
\hline $0-12$ & 12 & 5,9 \\
\hline $13-24$ & 46 & 22,9 \\
\hline $25-36$ & 52 & 25,9 \\
\hline $37-48$ & 47 & 23,4 \\
\hline $49-60$ & 39 & 19,4 \\
\hline $61-71$ & 5 & 2,5 \\
\hline
\end{tabular}

* Oito entrevistados não informaram a renda; ** Um dado não registrado

Fonte: elaborado pelos autores com dados na pesquisa.
Quando os pais foram perguntados sobre a recomendação de não usar o flúor nos dentifrícios, 164 $(81,2 \%)$ responderam que não obtiveram essa recomendação, e 38 (18,8\%) responderam afirmativamente para essa recomendação. A Tabela 2 mostra as atitudes dos pais em relação à higiene dos filhos.

Tabela 2 - Atitudes dos pais em relação à higiene dos filhos, Pelotas, $2013(n=202)$

\begin{tabular}{l|r|r}
\hline \multicolumn{1}{c|}{ Variáveis } & $\mathrm{N}$ & $\%$ \\
\hline Quem realiza a escovação da criança* & & \\
Criança & 24 & 12,2 \\
Ajuda de um adulto & 94 & 47,7 \\
Adulto & 79 & 40,1 \\
Quem coloca a pasta de dente na escova** & & \\
Criança & 22 & 10,9 \\
Adulto & 176 & 87,1 \\
Início do uso do dentifrício fluoretado & & \\
1 ano ou menos & 87 & 43,1 \\
Mais 1 ano & 44 & 21,8 \\
Não lembra & 71 & 35,1 \\
Quantidade de flúor apontada no folheto & & \\
Grão de arroz & 53 & 26,2 \\
Técnica transversal & 83 & 41,0 \\
Técnica longitudinal & 65 & 32,1 \\
Outros & 1 & 0,5 \\
Quantidade de flúor no dentifrício que compra & & \\
Menor que 1.100 ppm & 40 & 19,8 \\
Convencional (1.100 ppm) & 125 & 61,9 \\
Não foi possível identificar & 37 & 18,3 \\
\hline * Cinco dados perdidos ** Quatro dados perdidos
\end{tabular}

A Figura 1 mostra a compilação dos dados coletados quanto aos critérios que motivam os pais na escolha do dentifrício para a criança.

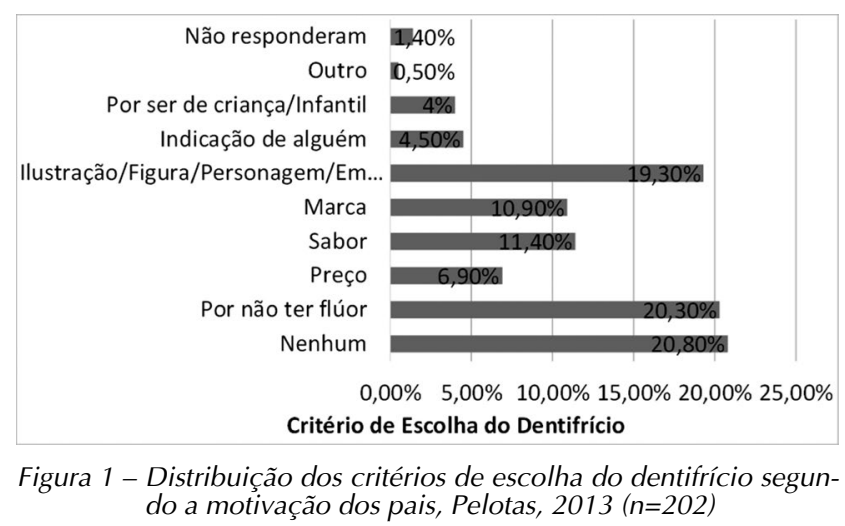

A Figura 2 apresenta a distribuição da amostra de acordo com a resposta à pergunta sobre a função do flúor no dentifrício. 


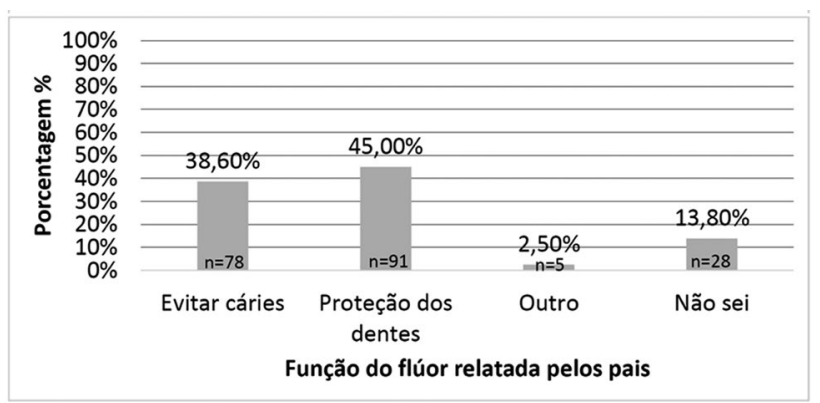

Figura 2 - Resposta dos pais com relação à finalidade do flúor, Pelotas, $2013(n=202)$

\section{Discussão}

A educação em saúde é uma das formas mais importantes para a prevenção de doenças. A grande maioria das doenças bucais é passível de prevenção. Por isso, investigar a atitude e o conhecimento da população a respeito das orientações mais corretas sobre os cuidados bucais, como quantidade e tipo de dentifrício e necessidade de expectoração para cada faixa etária, torna-se extremamente relevante, pois é por meio dessa análise que é possível propor melhorias com base nas deficiências encontradas.

$\mathrm{O}$ cuidado para não utilizar o dentifrício fluoretado em crianças menores de cinco anos de idade ${ }^{22}$, devido à ingestão de flúor e risco de desenvolvimento de fluorose dentária ${ }^{22}$, não é mais indicado. Porém, essa informação ainda pode ser motivo de confusão, não somente para a população em geral como entre os cirurgiões-dentistas e outros profissionais de saúde.

A nova recomendação indica que o uso dos dentifrícios fluoretados deve ser administrado em todas as faixas etárias, a partir do primeiro dente decíduo. $\mathrm{O}$ cuidado deve ser com relação à dose do produto para as diferentes faixas etárias ${ }^{4,6,7}$. A orientação atual recomenda que para bebês com até oito dentes na boca ou com menos de $10 \mathrm{~kg}$ deve-se colocar a quantidade equivalente à metade de um grão de arroz cru $(0,05 \mathrm{~g})$; bebês e crianças que não sabem cuspir e com mais de $10 \mathrm{~kg}$, colocar a quantidade equivalente a um grão de arroz $(0,1 \mathrm{~g})$; para crianças que sabem cuspir, até os seis anos, a quantidade deve ser equivalente a um grão de ervilha $(0,3 \mathrm{~g})^{6}$.

Com base nos achados deste estudo, pode-se inferir que essa orientação, na população pesquisada, ainda não está difundida, uma vez que $73,1 \%$ dos pais estão indicando a técnica transversal ou longitudinal para filhos com idades até cinco anos. É preciso chamar a atenção para esse achado, pois as crianças ainda não apresentam o reflexo expectorante adequado ${ }^{13}$ nessa fase, e o uso exagerado do produto pode acarretar na ingestão crônica de flúor, ocasionando a fluorose dentária na dentição permanente da criança, pois as coroas dentárias permanentes ainda estão em formação ${ }^{23}$.

Entre os pais entrevistados, $87,1 \%$ são responsáveis pela colocação do dentifrício nas escovas dentais, sendo esse um aspecto importante, pois quando é a criança que realiza essa etapa, há uma tendência a adicionar dentifrício em grandes quantidades, principalmente se tiverem gosto agradável. Assim, pelo fato de serem os responsáveis por colocar o produto nas escovas, esperar-se-ia maior controle. No entanto, ao observarmos a indicação da ilustração que melhor representava a quantidade de dentifrícios utilizada pelos pais, a maioria indicou doses acima do normal. Achado similar foi encontrado por Huebener et al. ${ }^{24}$, que ao mensurarem a quantidade de dentifrício colocada nas escovas dentais por pais norte-americanos de crianças de doze a 71 meses de idade, encontraram que a maioria utilizava o produto em quantidade acima do recomendado.

Ainda com relação aos hábitos de higiene da criança, a escovação é realizada pelo adulto ou pela criança com ajuda do adulto em $87,8 \%$. No entanto, mesmo em porcentagem mais baixa, $12,2 \%$ das crianças entre um e 65 anos estão escovando os dentes sozinhas, o que é uma informação preocupante, pois crianças dessa faixa etária não têm destreza manual para uma efetiva higiene bucal, o que acarreta em uma limpeza insuficiente dos dentes e maior risco de doenças bucais. Por isso a importância de os pais serem os responsáveis pela escovação dos dentes dos filhos. Com relação ao início da escovação com o dentifrício fluoretado, apenas $43,1 \%$ relatou que começou antes de um ano de idade, sendo outro fator a ser considerado, pois a nova indicação é que se comece desde o surgimento do primeiro dente decíduo, ou seja, por volta dos seis ou oito meses de idade ${ }^{4,6,7}$.

Além disso, aproximadamente $20 \%$ dos pais estão escolhendo dentifrícios sem flúor ou em quantidades inferiores à recomendada. É preciso chamar a atenção para esse dado, uma vez que existem evidências na literatura de que o dentifrício em concentrações inferiores a $1.100 \mathrm{ppm}$ de flúor aumentam significativamente o risco de cárie em dentes decíduos, e não previne a fluorose esteticamente questionável em dentes anteriores permanentes ${ }^{4,22,25,26}$. A fluorose não pode ser considerada como um problema de saúde pública ${ }^{27}$. Um estudo realizado em Pelotas revelou uma prevalência de $8,6 \%$, sendo $6,85 \%$ relacionada a graus muito leve ou leve, estes achados não apresentam preocupação a nível estético ${ }^{27}$.

A escovação dentária com dentifrício fluoretado é considerada um dos métodos mais eficientes na prevenção da cárie dentária, pois desorganiza o biofilme bacteriano e expõe flúor na cavidade bucal de forma regular ${ }^{28}$. Quando os pais foram perguntados sobre a função do flúor, mais de $80 \%$ indicaram que ele serve para prevenir cáries ou para proteger os dentes, o que é uma constatação positiva, pois a maioria dos pais está informada sobre os benefícios do flúor para a saúde bucal ${ }^{16,17}$. No entanto, parece que os pais não reconhecem os riscos e a importância da quantidade/dose do produto. Imagina-se que por optarem por um produto infantil, eles entendem que já existe um cuidado na formulação, sendo desnecessária a preocupação com a quantidade. 
Verificou-se uma variedade de critérios para a escolha dos pais pelo dentifrício, os critérios mais citados foram o fato de não ter flúor e a ilustração/ imagem da embalagem. A escolha deveria ser norteada pela presença do flúor, uma vez que reconhecem a sua importância. Essa revelação confirma a carência de informação sobre as novas recomendações do uso dos dentifrícios para crianças.

A escolha baseada na imagem/ilustração denota o desconhecimento referente às diferentes formulações presentes no mercado de dentifrícios infantis. Há no mercado uma ampla variedade de produtos exclusivos para crianças ${ }^{19}$. A linha infantil, de modo geral, busca sempre atrair a atenção com embalagens coloridas e diferentes apresentações, decoradas com vários personagens e, muitas vezes, o próprio dentifrício é colorido e aromatizado, contribuindo para uma maior ingestão do produto. Há muitos sabores (menta, tutti-frutti), cores (azul, verde, rosa) e consistências (gel ou pasta). Com base nisso, constatou-se que não há uma padronização para a compra do produto, e existe a necessidade de maior esclarecimento dos pais a respeito dos dentifrícios infantis para a escolha correta desses produtos.

Em relação a pesquisas que utilizam entrevistas, é preciso ter cautela com os resultados obtidos, pois, de acordo com Martins et al. ${ }^{29}$ (2011), os pais apresentam um comportamento mais cuidadoso durante entrevistas por estarem sendo submetidos a uma pesquisa, esse aspecto é chamado de viés de cortesia $^{30}$. Portanto, é preciso ter cuidado na interpretação dos resultados, uma vez que os pais podem ter respondido as perguntas de acordo com o que $o$ profissional esperasse ouvir, introduzindo um erro sistemático de informação.

A entrevista foi conduzida no maior supermercado central da cidade, que é referência para os diferentes bairros do município. Esse ambiente, até final do ano de 2013, previamente à inauguração do shopping da cidade, era um estabelecimento que oferecia produtos alimentícios e servia como ponto de compras de utensílios gerais para as famílias, ou seja, era um local frequentado por muitas famílias. Por ter essa característica central, associada à oferta de diferentes produtos, pode-se considerar que atinge uma parcela heterogênea da população, o que pode ser constatado pela renda familiar coletada, que variou de um salário mínimo a 21 vezes esse valor.

Além disso, a escolha de um supermercado para a coleta de dados ocorreu por conveniência, uma vez que nesses locais os pais poderiam mostrar qual era o dentifrício que costumavam comprar e, assim, era possível identificarmos se era sem flúor, com baixo teor ou convencional, minimizando o viés de memória. No entanto, o uso de um único local para a coleta de dados representa uma limitação deste trabalho, bem como o uso de uma amostra por conveniência, pois não podemos extrapolar esses dados para toda a população da cidade.
Além dessa limitação, este é um estudo transversal e, portanto, nenhuma relação de causalidade pode ser determinada. Somado a isso, este estudo utilizou como ferramenta uma entrevista, com perguntas semiestruturadas que podem induzir a uma resposta que é socialmente desejável, e desta forma temos que interpretar os dados sempre com cautela.

Mesmo diante das limitações, é possível verificar a necessidade de se investir em mais educação em saúde bucal para os pais e responsáveis de crianças pré-escolares, uma vez que está claro na literatura os benefícios dos dentifrícios fluoretados para a prevenção da cárie dentária, desde que usado corretamente. É de extrema relevância que profissionais da área da saúde e pais conheçam as novas recomendações e, para isso, é necessária a elaboração de novas políticas públicas em saúde em relação à utilização correta dos dentifrícios fluoretados.

\section{Conclusão}

Com base nos achados do presente estudo, verifica-se que os pais das crianças são portadores de conhecimentos sobre o flúor e sua finalidade, porém, não utilizam a dose recomendada de acordo com a idade da criança e não há conscientização quanto à motivação para a escolha do dentifrício infantil. Assim, há necessidade de investir em educação e informação a respeito dos dentifrícios para as crianças e suas indicações.

\section{Abstract}

Objective: To assess the knowledge of parents on fluoride and dentifrices, as well as their use, and the reason for choosing them. Subjects and method: Cross-sectional study with data collected through interviews with parents of children aged 1-65 months, addressing socioeconomic issues, their knowledge on fluoride, the reason for dentifrice choice, the amount used, and child's oral hygiene habits. Data were analyzed through descriptive statistics. Results: 202 parents were interviewed. The mean age of children participating in the study was 35.6 months $( \pm 15.06)$. Parents reported the following criteria for buying the dentifrice: absence of fluoride $(20.3 \%)$, price $(6.9 \%)$, flavor $(11.4 \%)$, brand (10.9\%), and $20.8 \%$ of parents reported no criterion. As for introducing fluoride dentifrice for the children, $43.1 \%$ of parents said that it occurred in up to one year, $21.8 \%$ later than one year, and $35.1 \%$ did not remember. Regarding the amount of dentifrice used, $32.2 \%$ of parents indicated using the longitudinal technique, $41.1 \%$ used the cross-sectional technique, $26.2 \%$ used the rice grain technique, and $5 \%$ did not respond. As for performing oral hygiene alone or with some type of help, in $12.2 \%$ of cases it is performed only by the children, while $47.7 \%$ of cases involves the help of an adult, and in $40.1 \%$ of cases it is performed only by the adult. Conclusion: Parents have knowledge on fluoride and its purpose, but do not use the recommended dose according to age, and there is no awareness regarding 
the reason for choosing the dentifrice. Thus, investments in education on dentifrices are required for an informed choice and proper use.

Keywords: Fluoride. Preschoolers. Dentifrices.

\section{Referências}

1. Brasil. Ministério da Saúde. Secretaria de Atenção à Saúde. Departamento de Atenção Básica. Saúde Bucal. Brasília: Ministério da Saúde; 2008. 92p. (Série A. Normas e Manuais Técnicos) (Cadernos de Atenção Básica; 17).

2. Brasil. Ministério da Saúde. Secretaria de Atenção à Saúde. Departamento de Atenção Básica. Pesquisa Nacional de Saúde Bucal SB Brasil 2010: Resultados Principais. Brasília: Ministério da Saúde; 2011. 92p. (Série C. Projetos, Programas e Relatórios Saúde).

3. Botelho JN, Cury AA, Silva WJ, Tenuta LM, Cury JA. The effect of fluoride toothpaste on root dentine demineralization progression: a pilot study. Braz Oral Res 2014; 28:1-5.

4. Cury JA, Tenuta LM. Evidence-based recommendation on toothpaste use. Braz Oral Res 2014; 28:1-7.

5. Maguire A. ADA clinical recommendations on topical fluoride for caries prevention. Evid Based Dent 2014; 15:38-9.

6. Oliveira BH, Santos APP, Nadanovsky, P. Use of fluoride toothpaste by preschoolers: what pediatricians should know? Res Ped 2012; 2(2):12-9.

7. World Health Organization (WHO). Inadequate or excess fluoride. World Health Organization, Geneva, Switzerland: Document Production Services; 2010.

8. Lima YB, Cury JA. Fluoride intake by children from water and dentifrice. Rev Saude Publica 2001; 35(6):576-81.

9. Sanglard-peixot LO, Zardetto CGC, Wanderley MT, Corrêa MSNP. Comparative analysys of dentifrices for children, available in brazilian market. JBP Rev Ibero-am Odontopediatr Bebê 2004; 7(37):273-81.

10. Pessan JP, Silva SM, Buzalaf MA. Evaluation of the total fluoride intake of 4-7-year-old children from diet and dentifrice. J Appl Oral Scien: Rev FOB 2003; 11(2):150-6.

11. Damião LS, Lopes PP, Castro AM, Oliveira FS. Use of fluoride dentifrice and toothbrushing habits in children aged 6 to 36 months. Rev Odontol Bras Central 2010; 19(51):295-300.

12. Zohoori FV, Buzalaf MA, Cardoso CA, Olympio KP, Levy FM, Grizzo LT, et al. Total fluoride intake and excretion in children up to 4 years of age living in fluoridated and non-fluoridated areas. Eur J Oral Sci 2013; 121(5):457-64.

13. Oliveira MJ, Paiva SM, Martins LH, Ramos-Jorge ML, Lima YB, Cury JA. Fluoride intake by children at risk for the development of dental fluorosis: comparison of regular dentifrices and flavoured dentifrices for children. Caries Res 2007; 41(6):460-6.

14. Chedid AJ, Guedes-Pinto A, Narvai PC, Forni TI, Bonecker M. Flúor - uso interno. In: Guedes-Pinto A. Odontopediatria. 8. ed. São Paulo: Santos; 2010. p. 417-68.

15. DenBesten PK. Biological mechanisms of dental fluorosis relevant to the use of fluoride supplements. Com Dent Oral Epidemiol 1999; 27(1):41-7.

16. Dincer E, Ligouri AL, Rayman S, Rivera A. Parental perceptions about children's oral health care and toothpaste in New York City neighborhoods. N Y State J 2009; 75(2):44-8.

17. Naidu RS, Davis L. Parents' views on factors influencing the dental health of Trinidadian pre-school children. Com Dent Health 2008; 25(1):44-9.
18. Bennadi D, Kshetrimayum N, Sibyl S, Reddy CV. Toothpaste utilization profiles among preschool children. J Clin Diagn Res 2014; 8(3):212-5.

19. Basch CH, Hammond R, Guinta A, Rajan S, Basch CE. Advertising of toothpaste in parenting magazines. J Com Health $2013 ; 38(5): 911-4$.

20. Lima NHS, Martins CC, Paiva SM. Apresentação comercial de dentifrícios convencionais e infantis presentes no mercado brasileiro. Pesq Bras Odontoped Clin Int 2005; 5(2):141-9.

21. Lima FG, Lund RG, Justino LM, Demarco FF, Del Pino FA, Ferreira R. Twenty-four months of external control of fluoride levels in the public water supply in Pelotas, Rio Grande do Sul, Brazil. Cad Saude Publica 2004; 20(2):422-9.

22. Santos AP, Oliveira BH, Nadanovsky P. Effects of low and standard fluoride toothpastes on caries and fluorosis: systematic review and meta-analysis. Caries Res 2013; 47(5):382-90.

23. Martins CC, Paiva SM, Lima-Arsati YB, Ramos-Jorge ML, Cury JA. Prospective study of the association between fluoride intake and dental fluorosis in permanent teeth. Caries Res 2008; 42(2):125-33.

24. Huebner CE, Thomas A, Scott J, Lin JY. Parents' interpretation of instructions to control the dose of fluoridated toothpaste used with young children. Ped Dent 2013; 35(3):262-6.

25. Oliveira MJ, Martins CC, Paiva SM, Tenuta LM, Cury JA Estimated fluoride doses from toothpastes should be based on total soluble fluoride. Int $J$ Environ Res Public Health 2013; 10(11):5726-36

26. Walsh T, Worthington HV, Glenny AM, Appelbe P, Marinho VC, Shi X. Fluoride toothpastes of different concentrations for preventing dental caries in children and adolescents. The Cochrane database of systematic reviews 2010: 20 (1): Cd007868.

27. Azevedo MS, Goettems ML, Torriani DD, Demarco FF. Factors associated with dental fluorosis in school children in southern Brazil: a cross-sectional study Braz Oral Res [ periódico online] 2014 [ citado 2014 maio]; 28 (1): [telas]. Disponível em URL: http://www.ncbi.nlm.nih.gov/pubmed/24878674.

28. Chedid AJ, Cury JA. O uso de fluoretos em odontopediatria fundamentado em evidências. In: Coutinho L, Bonecker, M. Odontopediatria para pediatria. 1 ed. São Paulo: Atheneu; 2013.p. 419-30.

29. Martins CC, Oliveira MJ, Pordeus IA, Cury JA, Paiva SM. Association between socioeconomic factors and the choice of dentifrice and fluoride intake by children. Int J Environ Res Public Health 2011; 8(11):4284-99.

30. León FR, Lundgren R, Huapaya A, Sinai I, Jennings V. Challenging the courtesy bias interpretation of favorable clients' perceptions of family planning delivery. Eval. Rev. 2007; 31(1):24-42.

\section{Endereço para correspondência:}

Marina Sousa Azevedo

Departamento de Odontologia Social e Preventiva

Rua Gonçalves Chaves 457, $7^{\circ}$ andar, sala 704

96015-560 Pelotas, RS

Telefone: 32224305- ramal 127

E-mail: marinasazevedo@hotmail.com

Recebido: 12/0615. Aceito: 12/08/15. 